\title{
Environmental stress maintains trioecy in nematode worms
}

\author{
Ashlyn G. Anderson ${ }^{1,2}$, Louis T. Bubrig ${ }^{1}$, Janna L. Fierst ${ }^{1, *}$ \\ 1 Department of Biological Sciences, The University of Alabama, Tuscaloosa AL 35487-0344 \\ 2 Current address: Department of Biostatistics, University of Florida, Gainesville FL \\ 32611-7450
}

\section{* Author for correspondence, jlfierst@ua.edu}

\begin{abstract}
Sex is determined by chromosomes in mammals but it can be influenced by the environment in many worms, crustaceans and vertebrates. Despite this, there is little understanding of the relationship between ecology and the evolution of sexual systems. The nematode Auanema freiburgensis has a unique sex determination system in which individuals carrying one $\mathrm{X}$ chromosome develop into males while XX individuals develop into females in stress-free environments and self-fertile hermaphrodites in stressful environments. Theory predicts that trioecious populations with coexisting males, females and hermaphrodites should be unstable intermediates in evolutionary transitions between mating systems. In this article we study a mathematical model of reproductive evolution based on the unique life history and sex determination of $A$. freiburgensis. We develop the model in two scenarios, one where the relative production of hermaphrodites and females is entirely dependent on the environment and one based on empirical measurements of a population that displays incomplete, 'leaky' environmental dependence. In the first scenario environmental conditions can push the population along an evolutionary continuum and result
\end{abstract}


in the stable maintenance of multiple reproductive systems. The second 'leaky' scenario results in the maintenance of three sexes for all environmental conditions. Theoretical investigations of reproductive system transitions have focused on the evolutionary costs and benefits of sex. Here, we show that the flexible sex determination system of $A$. freiburgensis may contribute to population-level resilience in the microscopic nematode's patchy, ephemeral natural habitat. Our results demonstrate that life history, ecology and environment may play defining roles in the evolution of sexual systems.

\section{Introduction}

The

\section{q}

of many different habitat types which can select for a diverse array of life history strategies. This

may select for mating systems that best take advantage of their sexes' particular strengths.

For instance, males and females are particularly suited for outcrossing because they cannot fertilize themselves. Outcrossing has many uses from promoting recombination to remove or slow the spread of deleterious alleles (Bell, 1982; Charlesworth and Charlesworth, 1987; Smith, 1978) and creating beneficial genetic combinations that facilitate adaptation (Crow, 1992; Smith, 1978). Outcrossing can therefore be a useful strategy for responding to ecological challenges. C. elegans outcrossing rates increase in response to starvation (Morran et al., 2009a), increased mutation load and adaptation to a new environment (Morran et al., 2009b), and coevolution with parasites (Morran et al., 2011). Males and females, being obligate outcrossers, guarantee these benefits. On 
the other hand, males and females can often be poor colonizers. Individuals must arrive at the same time and place and in the right sex ratios to successfully colonize a new habitat.

Self-compatible hermaphrodites are better suited for dispersal and colonization because they can fertilize themselves independent of the arrival of others (Baker, 1955, 1967). Dispersal is also a useful strategy for responding to ecological challenges. It enables organisms to leave degraded habitats and avoid breeding with close relatives which depresses fitness (Charlesworth and Willis, 2009) and slows the production of new genetic variants (Wright, 1933). Certain habitat types, like rotting organic matter, require regular dispersal between patches as patches degrade. For instance, nematodes in the order Rhabditida exploit such habitats and even have a designated dispersal stage called the dauer (Felix and Braendle, 2010; Kiontke et al., 2011; Fig. 2a) which is induced by environment stress including increasing temperature, increasing population density, or decreasing food levels (Ailion and Thomas, 2000; Cassada and Russell, 1975; Golden and Riddle, 1984). Dauers attach to invertebrate hosts to leave stressful patches and resume development on new patches (Golden and Riddle, 1984; Kiontke et al., 2011; Poinar, 1983). Lineages that live in similarly patchy habitats may find hermaphrodites useful for their dispersal capabilities despite their drawback, namely their lack of obligate outcrossing.

Lineages may employ mating systems that balance the comparative need for outcrossing versus colonizing, although a mating system with all three sexes could have the best of both worlds. Despite this, species employing three sexes had not been found and trioecy was long thought to be a temporary, transitory mating system (Charlesworth, 1984; Lande and Schemske, 1985). Previous mathematical models have failed to find stable trioecious equilibra (Chasnov, 2010; Gregorius et al., 1983; Pannell, 2008; Wolf and Takebayashi, 2004; Fig. 1a). These theoretical predictions are now at odds with accumulating empirical evidence for trioecy in crustaceans (Sassaman and Weeks, 1993), plants (Mirski et al., 2017), and nematodes (Chaudhuri et al., 2015; Kanzaki et al., 2017). Auanema is a recently described nematode genus with two trioecious species, A. rhodensis and A. freiburgensis (Kanzaki et al., 2017) and is distantly related to the trioecious Heterorhabditis sp. (Strauch et al., 1994). 
In this article, we develop a new mathematical model to study how the flexible sex determination system of $A$. freiburgensis (Chaudhuri et al., 2011; Fig. 2b) can generate and maintain all three sexes. While there is a body of work studying the molecular underpinnings of reproductive systems in nematodes (Berenson and Baird, 2018; Guo et al., 2009; Hill et al., 2006; Yin and Haag, 2019), there is little understanding of the ecological factors that push some species towards androdioecy while others maintain dioecy or trioecy (Fig. 1b). Our model uses empirical species data to show that A. freiburgensis may employ trioecy to guarantee outcrossing and dispersal, contributing to environmental resilience at a population level and causing the persistence of this rare mating system. Dependence of life history on environment may therefore play a key role in the evolution of sexual systems.

\section{Materials and Methods}

\section{Biology of $A$. freiburgensis}

In A. freiburgensis, mothers sense environmental stress, which may be increasing temperature, increasing population density or decreasing food (Ailion and Thomas, 2000; Cassada and Russell, 1975; Golden and Riddle, 1984). As stress increases, mothers induce more of their offspring to become developmentally-arrested, non-feeding dauer. Unlike other Rhabditids, Auanema spp. use the dauer stage for part of their sex determination, making it a hybrid chromosomal/environmental system (Chaudhuri et al., 2011; Zuco et al., 2018). A. freiburgensis males have one copy of the sex chromosome (XO) and nonmales have two (XX) (Kanzaki et al., 2017). A. freiburgensis XX nonmales can either become adult females or hermaphrodites depending on whether or not they pass through the dauer stage, a decision induced by their mother after sensing stress (Fig. 1b). XX adults that do not go through dauer are always female (Chaudhuri et al., 2011) while those that develop via dauer are always hermaphrodites (Zuco et al., 2018). Males cannot pass through dauer at all and thus their development is not thought to be affected by environmental stress (Chaudhuri et al., 2011; Fig. 1b). Hermaphrodites are self-compatible and can be fertilized by 
males, but hermaphrodites cannot fertilize females or other hermaphrodites.

The sex ratios produced by Auanema spp. are highly skewed due to atypical gamete formation and spermatogenesis (Fig. 3; Shakes et al., 2011; Tandonnet et al., 2018). Meiosis has not been characterized in $A$. freiburgensis but it has for its close relative $A$. rhodensis and we assume the process in A. freiburgensis is similar. Hermaphrodites produce diplo-X sperm and nullo-X eggs resulting in male progeny when hermaphrodites are outcrossed (Table 1-2; Tandonnet et al., 2018). Males are produced at a reduced rate from male-female crosses and hermaphrodite selfing (Table 1-2). Two sets of sex ratios were compiled for this model: Model 1, in which stress perfectly explains XX phenotype and Model 2 based on empirical data of a 'leaky' environmental dependence (Zuco et al., 2018; A. Pires-daSilva, personal communication).

\section{Model 1}

We first build a model in which nonmale phenotype is strictly determined by stress, $s$ (Table 1). When no stress is present, $s=0$ and all $\mathrm{XX}$ nonmale progeny become females. When $s=1$, the worms experience maximum stress and all XX nonmales become hermaphrodites. Since dauer-inducing stress is not binary but a continuous scale (Cassada and Russell, 1975), we assume that as stress increases from $s=0$ to $s=1$, the frequency of hermaphrodites increases and the frequency of females decreases in a linear relationship (SFig. 2). The mutational path from dioecy to self-fertility in nematodes is likely short (Baldi et al., 2009). For example, in the dioecious $C$. remanei just two mutations, one lowering transcription levels of the gene tra-2 to permit sperm production and one increasing transcription of the sperm activation protein SWM-1, are required for females to generate self-sperm (Baldi et al., 2009). Accordingly, we assume these mutations occur frequently in natural populations and focus here on studying the conditions that maintain trioecy after these mutations have arisen.

For the resulting model, let the frequency of females be $P$, hermaphrodites be $Q$, and males be $R$. Not all individuals will find a male mate, so $\theta_{F}$ and $\theta_{H}$ represent the probability that a female and hermaphrodite are unable to find a male mate, respectively. Therefore, the proportion of 
females that reproduce is $\left(1-\theta_{F}\right) P$. The proportion of hermaphrodites that self is $\theta_{H} Q$.

The progeny of selfed hermaphrodites are expected to have some degree of fitness cost due to inbreeding depression. Let $d$ be equal to the relative fitness cost when compared to outcrossed progeny, such that $(1-d) Q$ is the proportion of selfed progeny that are viable. So that all frequencies sum to unity, we must denote a normalizing variable $w$. For each generation $n$, we have the following model:

$$
\begin{aligned}
& w P_{n}=(1-s)\left(0.82\left(1-\theta_{F}\right) P_{n-1}+0.913(1-d) \theta_{F} Q_{n-1}\right) \\
& w Q_{n}=s\left(0.82\left(1-\theta_{F}\right) P_{n-1}+0.863(1-d) \theta_{H} Q_{n-1}\right) \\
& w R_{n}=\left(1-\theta_{H}\right) Q_{n-1}+0.18\left(1-\theta_{F}\right) P_{n-1}+0.087(1-s)(1-d) \theta_{H} Q_{n-1}+0.136 s(1-d) \theta_{H} Q_{n-1}
\end{aligned}
$$

where the normalizing equation is:

$$
w=P_{n}+Q_{n}+R_{n}
$$

For each run of the model, the starting frequencies of the three sexes $\left(P_{0}, Q_{0}\right.$, and $\left.R_{0}\right)$ sum to unity but are otherwise randomly chosen. We explored constant intermediate stress by randomly assigning a value between $s=0$ and $s=1$ and maintaining this across all future generations. To model populations experiencing fluctuating stress we assigned $s$ a new value drawn from a standard uniform distribution $U(0,1)$ in each generation (SFig. 3). We assumed that males are always present at a low level in these populations and studied the influence of parameters on the relative proportions of females and hermaphrodites by iterating the stress parameter $s$, the inbreeding coefficient $d$, the difficulty of finding a mate for females $\Theta_{F}$ and the difficulty of finding a mate for hermaphrodites $\Theta_{H}$ across the range of possible values .

\section{Model 2}

The second model incorporates empirical sex ratio data measured in laboratory populations of $A$. freiburgensis (Table 2). Stress has been shown to have no effect on the sex determination of 
the progeny of a male-female cross, possibly because the laboratory strain of $A$. freburgensis is hyper-sensitive to crowding and is constantly stressed at the densities needed for reproduction (S. Tandonnet, personal communication). Additionally, a 'leak' is seen in hermaphrodite selfing such that a small proportion of hermaphrodites are produced when there is no stress, and a small proportion of females are produced when there is stress (A. Pires-daSilva, personal communication). We still assume a linear relationship between environmental stress $(0>s>1)$ and XX nonmale phenotype, though hermaphrodites are overrepresented in low stress compared to Model 1 (SFig. 4).

Hermaphrodites will be produced under any amount of environmental stress; when $s=0$, hermaphrodites have a frequency of $\approx 0.4$. However, the frequency of females will mirror Model 1 and continue to decrease as stress increases:

$$
\begin{aligned}
& w P_{n}=0.911(1-s)(1-d) \theta_{H} Q_{n-1}+0.062 s(1-d) \theta_{H} Q_{n-1} \\
& w Q_{n}=0.82\left(1-\theta_{F}\right) P_{n-1}+0.801 s(1-d) \theta_{H} Q_{n-1}+0.002(1-s)(1-d) \theta_{H} Q_{n-1} \\
& w R_{n}=\left(1-\theta_{H}\right) Q_{n-1}+0.18\left(1-\theta_{F}\right) P_{n-1}+0.087(1-s)(1-d) \theta_{H} Q_{n-1}+0.136 s(1-d) \theta_{H} Q_{n-1}
\end{aligned}
$$

where the normalizing equation is:

$$
w=P_{n}+Q_{n}+R_{n}
$$

We also modeled a semi-random stress distribution that more accurately represents natural populations of $A$. freiburgensis. A. freiburgensis was isolated from dung, an ephemeral patchy habitat, and the closely-related A. rhodensis was isolated using invertebrates as bait to pick up waiting dauers (Kanzaki et al., 2017), so we believe Auanema spp. act like typical Rhabditids. We assume here that in small ephemeral patches, stress will continually increase as the patch degrades. Once the patch is too stressful, A. freiburgensis dauers would likely abandon the patch and disperse to new lower-stress patches. Therefore, environmental stress may approximate a sawtooth pattern (SFig. 5). To represent stochastic patch degradation $s_{n+1}=s_{n}+x^{2}+y^{2}(1-x)$ 
where $x$ and $y$ are drawn from the standard uniform distribution $U(0,1), s_{0}=x$ and $y$ is redrawn each generation. If $s>0.7$ (an arbitrary dispersal threshold) both $x$ and $y$ are redrawn from the standard normal distribution $U(0,1)$ to represent dispersal to a fresh patch. The speed at which the patch degrades is stochastic, as well as the number of generations per patch and the initial stress level of each new patch. For Model 2 we also assumed that males are always present at a low level and studied the influence of parameters on the relative proportions of females and hermaphrodites by iterating across the range of possible values for stress $s$, inbreeding $d$, the difficulty of finding a mate for females $\Theta_{F}$ and the difficulty of finding a mate for hermaphrodites $\Theta_{H}$

\section{Results}

\section{Model 1}

When there is no stress $(s=0)$, the population is composed of only females and males. Any hermaphrodites present at generation $n=0$ are immediately lost and do not return, as no XX nonmales can become hermaphrodites when $s=0$ (Fig. 4a). When there is constant stress $(s=1)$, females drop out of the model and the population is composed of only hermaphrodites and males (Fig. 4b). At constant intermediate stress $(s=0.5)$ males, females and hermaphrodites are maintained in the population (Fig. 4c). When the idealized model is run with environmental stress $s$ that randomly fluctuates with each new generation, all three sexes are maintained indefinitely regardless of starting proportions (Fig. 4d). A single hermaphrodite can invade a dioecious population and result in the maintenance of a trioecious population (SFig. 6).

The complex coefficients of the normalizing equation reduce to $w=P_{n}\left(1-\Theta_{F}\right)+Q_{n}(1-$ $\left.\Theta_{H}(d(1+0.001 s)-0.001 s)\right)$. Accordingly, the stress level $s$ has the largest influence on the proportion of $X X$ individuals that are female in the population (Fig. 5) with the inbreeding coefficient, $d$, exerting little influence. The relative difficulty of finding a mate for females $\Theta_{F}$ and for hermaphrodites $\Theta_{H}$ impact the relative proportions of females and hermaphrodites but $s$ 
determines the maximum and minimum values of these proportions (SFig 7-9).

\section{Model 2}

The 'leaky' sex ratio data of Model 2 results in the maintenance of trioecious populations for all scenarios including no stress $(s=0$; Fig. 6a), constant high stress $(s=1$; Fig. 6b), constant intermediate stress $(s=0.5$, Fig. $6 c)$ and fluctuating stress (Fig. 6d). A single hermaphrodite produces female, hermaphrodite and male offspring and can found a new trioecious population (Fig. 7). The sex determination system under Model 2 is complex and the relative proportion of females and hermaphrodites has nonlinear dependence on $s, d, \Theta_{F}$, and $\Theta_{H}$ (Fig. 8). The parameter $s$ exerts a large influence on the relative proportion of $\mathrm{XX}$ individuals that are female and determines the maximum proportions of females and hermaphrodites in the population (SFig $10-12)$

Discussion

The evolution of self-fertility affects important aspects of a species' genetics including the spectrum of mutational effects (Charlesworth, 2003) and molecular and developmental adaptation (Shimizu and Tsuchimatsu, 2015). The causes and consequences of reproductive evolution are thus important questions in biology. Previous authors have used mathematical models to demonstrate that trioecious systems should be temporary, transitive states (Chasnov, 2010; Gregorius et al., 1983; Pannell, 2008; Wolf and Takebayashi, 2004). In the work presented here, a trioecious system was supported by a mathematical model when the model incorporated empirically measured values from $A$. freiburgensis. Our model demonstrated that females, males and hermaphrodites co-exist in the population depending on the dynamics of environmental stress. Flexibility in sex determination and mating system may be critical for population resilience in patchy, resourcelimited environments.

In our first model, dependence on stress produced the entire range of reproductive modes 
observed in Rhabditid nematodes. When stress was absent the population was dioecious (Fig. 4a) and constant high stress pushed populations to androdioecy (Fig. 4b). Intermediate levels of stress, whether constant or fluctuating, resulted in the stable maintenance of trioecious populations (Fig. 4c-d). A single hermaphrodite, produced by a short mutational path in nematodes, could readily invade a dioecious population experiencing intermediate stress (Fig. 7).

Our second model, based on laboratory measurements of $A$. freiburgensis, resulted in trioecious populations for all tested parameter combinations. In these laboratory populations the female stress experience does not alter the sex ratio of her offspring. Relative to our first model the sex ratios of the second model were 'leaky' and environmental sex determination only occurred for offspring of hermaphrodites. This leaky system confined populations to trioecy. Hermaphrodites selfed to produce all three sexes in both stressful and stress-free conditions and a single hermaphrodite could readily found a new trioecious population.

In our first model, stress determined mating system transitions. A stress-free environment did not induce the formation of hermaphrodites at any appreciable level, collapsing the trioecious system down to a functionally dioecious system with males and females. In natural populations, deleterious mutations in hermaphrodite-forming genes could then knock hermaphrodites out of the species and solidify a true dioecious system. On the other hand, an environment of constant high stress caused XX individuals to become hermaphrodites, collapsing the system down to a functionally androdioecious one. Importantly, these alterations could also come about through changes in how the animal senses stress. For example, the laboratory strain of $A$. freiburgensis is thought to be hyper-sensitive to crowding and senses that it is always under stressful conditions in the lab environment. Similarly, a nematode strain could evolve to a stress-free state by becoming less sensitive to stressors; Caenorhabditis dauer formation can be induced with pheromones (Golden and Riddle, 1984) and there is natural variation among strains of C. elegans for pheromone response and dauer induction (Viney et al., 2003). Loss-of-function deletions in the chemoreceptor genes srg-36 and srg-37 appear to underlie part of this variation in C. elegans and the lab-adapted N2 strain is relatively less sensitive to dauer pheromones than wild-collected strains (Lee et al., 2019). 
Rather than being a temporary or transitional mating system (Charlesworth, 1984; Lande and Schemske, 1985), trioecy may be a stable adaptive strategy to reap the benefits of outcrossing and dispersal in the same organism, avoiding the drawbacks inherent in androdioecy and dioecy. Androdioecious nematodes have hermaphrodites which are superior colonizers but may suffer from mutation accumulation because they are not obligate outcrossers; though males can outcross with hermaphrodites, hermaphrodites can and will self-fertilize even in the presence of males. The outcrossing rate in many androdioecious species is lower than the selfing rate (Barriere and Felix, 2005; Charlesworth, 1984; Sivasundar and Hey, 2003). Dioecious nematodes on the other hand guarantee outcrossing but are not as successful at colonization because they lack hermaphrodites. Trioecy combines both strategies by having a self-fertilizing sex conducive to colonization while also having obligate outcrossing sexes conducive to maintaining genetic diversity within a patch. How the production and maintenance of the three sexes is controlled to best utilize their strengths is probably group specific, but the nematode $A$. freiburgensis appears to do this by tying the production of hermaphrodites to stress which induces the formation of a higher percentage of dauer larvae. New habitats can be colonized by self-fertile hermaphrodites, allowing for a first generation to be produced quickly and reliably. This first generation is born into a lower stress, resource-rich habitat and can produce obligate outcrossing sexes until environmental stress or inbreeding levels are too high and dispersal is necessary again.

The application of Baker's Law to metapopulations shows that the relative colonization advantage provided by self-compatible hermaphrodites depends on many parameters including rate of habitat decay and rate of colonization (Pannell and Barrett, 1998). Different habitats with different colonization and extinction rates might cause trioecy to collapse down to either dioecy or androdioecy depending on the comparative need for outcrossing versus colonizing. Differences in habitat and ecological context could at least in part explain the wide variety of mating systems seen in Rhabditida and in other such as crustaceans and plants.

One of the odd limitations of the nematode system is that while much is known about their underlying genetics and development, very little is known about nematode ecology (Felix and 
Braendle, 2010). This is in part due to a historical mis-identification of the model organism $C$. elegans' natural habitat that has only recently been corrected (Kiontke et al., 2011) as well as the primary focus on C. elegans' genetics and development (Brenner, 1974). C. elegans is ostensibly only one species out of many in Rhabditida, but its lack of good natural ecology data has affected research that uses C. elegans as a reference and a starting point, leaving our understanding of Rhabditid natural habitats and colonization severely lacking. The work we have presented here demonstrates that a complete understanding of the causes and consequences of reproductive evolution will require an integrated approach to studying the ecology, genetics and development of real organisms.

\section{Acknowledgments}

\section{Literature Cited}

Ailion, M. and J. H. Thomas, 2000. Dauer formation induced by high temperatures in Caenorhabditis elegans. Genetics 156:1047-1067.

Baker, H. G., 1955. Self-compatibility and establishment after 'long-distance' dispersal. Evolution 9:347-349.

— 1967. Support for Baker's law - as a rule. Evolution 21:853-856.

Baldi, C., S. Cho, and R. E. Ellis, 2009. Mutations in two independent pathways are sufficient to create hermaphroditic nematodes. Science 326:1002-1005.

Barriere, A. and M. Felix, 2005. High local genetic diversity and low outcrossing rate in Caenorhabditis elegans natural populations. Current Biology 15:1176-1184.

Bell, G., 1982. The Masterpiece of Nature. University of California Press, Los Angeles, CA.

Berenson, A. L. and S. E. Baird, 2018. Regulation of the sperm-to-oocyte transition in Caenorhabditis 
briggsae hermaphrodites by the Cbr-met-2 and Cbr-fem-3 genes. Molecular Reproduction and Development 85:532-542.

Blaxter, M. L., P. De Ley, J. R. Garey, L. X. Liu, P. Schedleman, A. Vierstraete, J. R. Vanfleteren, L. Y. Mackey, M. Dorris, L. M. Frisse, J. R. Vida, and W. K. Thomas, 1998. A molecular evolutionary framework for the phylum Nematoda. Nature 392:71-75.

Brenner, S., 1974. The genetics of Caenorhabditis elegans. Genetics 77:71-94.

Cassada, R. C. and R. L. Russell, 1975. The dauer larva, a post-embryonic developmental variant of the nematode Caenorhabditis elegans. Developmental Biology 46:326-342.

Charlesworth, D., 1984. Androdioecy and the evolution of dioecy. Biological Journal of the Linnean Society 22:333-348.

- 2003. Effects of inbreeding on the genetic diversity of populations. Philosophical Transactions of the Royal Society Series B 358:1051-1070.

Charlesworth, D. and B. Charlesworth, 1987. Inbreeding depression and its evolutionary consequences. Annual Review of Ecology Evolution and Systematics 18:237-268.

Charlesworth, D. and J. H. Willis, 2009. The genetics of inbreeding depression. Nature Reviews Genetics 10:783-796.

Chasnov, J. R., 2010. The evolution from females to hermaphrodites results in a sexual conflict over mating in androdioecious nematode worms and clam shrimp. Journal of Evolutionary Biology 23:539-556.

Chaudhuri, J., N. Bose, S. Tandonnet, S. Adams, G. Zuco, V. Kache, M. Parihar, S. H. von Reuss, F. C. Schroeder, and A. Pires-daSilva, 2015. Mating dynamics in a nematode with three sexes and its evolutionary implications. Scientific Reports 5:17676.

Chaudhuri, J., V. Kache, and A. Pires-daSilva, 2011. Regulation of sexual plasticity in a nematode that produces males, females, and hermaphrodites. Current Biology 21:1548-1551. 
Crow, J. F., 1992. An advantage of sexual reproduction in a rapidly changing environment. Journal of Heredity 83:169-173.

Felix, M. A. and C. Braendle, 2010. The natural history of Caenorhabditis elegans. Current Biology 20:R965-R969.

Golden, J. W. and D. L. Riddle, 1984. The Caenorhabditis elegans dauer larva: Developmental effects of pheromone, food and temperature. Developmental Biology 102:368-378.

Gregorius, H. R., M. D. Ross, and E. M. Gillet, 1983. Selection in plant populations of effectively infinite size V. Biallelic models of trioecy. Genetics 103:529-544.

Guo, Y., S. Lang, and R. E. Ellis, 2009. Indepdendent recruitment of F box genes to regulate hermaphrodite development during nematode evolution. Current Biology 19:1853-1860.

Hill, R. C., C. E. de Carvalho, J. Salogiannis, B. Schlager, D. Pilgrim, and E. S. Haag, 2006. Genetic flexibility in the convergent evolution of hermaphroditism in Caenorhabditis nematodes. Developmental Cell 10:531-538.

Kanzaki, N., K. Kiontke, R. Tanaka, Y. Hirooka, A. Schwarz, T. Muller-Reichert, J. Chaudhuri, and A. Pires-daSilva, 2017. Description of two three-gendered nematode species in the new genus Auanema (Rhabditina) that are models for reproductive mode evolution. Scientific Reports $7: 1-14$.

Kiontke, K. C., M. A. Felix, M. Ailion, M. V. Rockman, C. Braendle, J. B. Penigault, and D. H. A. Fitch, 2011. A phylogeny and molecular barcodes for Caenorhabditis, with numerous new species from rotting fruits. BMC Evolutionary Biology 11.

Lande, R. and D. W. Schemske, 1985. The evolution of self-fertilization and inbreeding depression in plants 1. Genetic Models. Evolution 39:24-40.

Lee, D., S. Zdraljevic, D. E. Cook, L. Frezal, J. Hsu, M. G. Sterken, J. A. G. Riksen, J. Wang, J. E. 
Kammenga, C. Braendle, M. Felix, F. C. Schroeder, and E. C. Andersen, 2019. Selection and gene flow shape niche-associated copy-number variation of pheromone receptor genes. BioRXiv .

Mirski, P., E. Brzosko, I. Jedrzejczyk, B. Ostrowiecka, and A. Wroblewska, 2017. Genetic structure of dioecious and trioecious Salix myrsinifolia populations at the border of geographic range. Tree Genetics and Genomes 13:15.

Morran, L. T., B. J. Cappy, J. L. Anderson, and P. C. Phillips, 2009a. Sexual partners for the stressed: Facultative outcrossing in the self-fertilizing nematodecaenorhabditis elegans. Evolution 63:14731482.

Morran, L. T., M. D. Parmenter, and P. C. Phillips, 2009b. Mutation load and rapid adaptation favour outcrossing over self-fertilization. Nature 462:350-352.

Morran, L. T., O. Schmidt, I. A. Gelarden, R. C. Parrish, and C. M. Lively, 2011. Running with the Red Queen: host-parasite coevolution selects for biparental sex. Science 6039:216-218.

Pannell, J. R., 2008. Consequences of inbreeding depression due to sex-linked loci for the maintenance of males and outcrossing in branchiopod crustaceans. Genetical Research Cambridge 90:73-84.

Pannell, J. R. and S. C. H. Barrett, 1998. Baker's law revisited: Reproductive assurance in a metapopulation. Evolution 52:657-668.

Poinar, G. O., 1983. The natural history of nematodes. Prentice Hall, Englewood Cliffs, NJ.

Sassaman, C. and S. C. Weeks, 1993. The genetic mechanism of sex determination in the conchostracan shrimp Eulimnadia texana. American Naturalist 141:314-328.

Shakes, D. C., B. J. Neva, H. Huynh, J. Chaudhuri, and A. Pires-daSilva, 2011. Asymmetric spermatocyte division as a mechanism for controlling sex ratios. Nature Communications P. 157. 
Shimizu, K. K. and T. Tsuchimatsu, 2015. Evolution of selfing: recurrent patterns in molecular adaptation. Annual Review of Ecology Evolution and Systematics 46:593-622.

Sivasundar, A. and J. Hey, 2003. Population genetics of Caenorhabditis elegans: the paradox of low polymorphism in a widespread species. Genetics 163:147-157.

Smith, J. M., 1978. The Evolution of Sex. Cambridge University Press, Cambridge.

Strauch, O., S. Stoessel, and R. U. Ehlers, 1994. Culture conditions define automictic or amphimictic reproduction in entomopathogenic Rhabditid nematodes of the genus Heterorhabditis. Fundamental and Applied Nematology 17:575-582.

Tandonnet, S., M. C. Farrell, G. D. Koutsovoulos, M. L. Blaxter, M. Parihar, P. L. Sadler, D. C. Shakes, and A. Pires-daSilva, 2018. Sex- and gamete-specific patterns of X chromosome segregation in a trioecious nematode. Current Biology 1:93-99.e3.

Viney, M. E., M. P. Gardner, and J. A. Jackson, 2003. Variation in Caenorhabditis elegans dauer larva formation. Development Growth and Differentiation 45:389-396.

Wolf, D. E. and N. Takebayashi, 2004. Pollen limitation and the evolution of androdioecy from dioecy. American Naturalist 163:122-137.

Wright, S., 1933. Inbreeding and homozygosis. Proceedings Of The National Academy Of Sciences 19:411-420.

Yin, D. and E. S. Haag, 2019. Evolution of sex ratio through gene loss. Proceedings Of The National Academy Of Sciences 116:12919-12924.

Zuco, G., V. Kache, P. Robles, J. Chaudhuri, B. Hill, C. Bateson, and A. Pires-daSilva, 2018. Sensory neurons control heritable adaptation to stress through germline reprogramming. BioRXiv . 
Table 1: In Model 1 the progeny of females and selfing hermaphrodites are female-biased in the absence of stress. When stress is present, these progeny go into dauer and become hermaphrodites. Male frequency is not impacted by stress. Data were compiled from Kanzaki et al., 2017; Shakes et al., 2011; Tandonnet et al., 2018; Zuco et al., 2018.

\begin{tabular}{|c|c|c|c|c|c|c|}
\hline & \multicolumn{3}{|c|}{ NO STRESS } & \multicolumn{3}{|c|}{ STRESS } \\
\hline & $\sigma^{\prime \prime}$ & $q^{\prime \prime}$ & o & o" & $q^{\prime \prime}$ & q \\
\hline 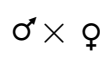 & $18 \%$ & $0 \%$ & $82 \%$ & $18 \%$ & $82 \%$ & $0 \%$ \\
\hline$\sigma^{\prime \prime} \times \sigma^{\prime \prime}$ & $100 \%$ & $0 \%$ & $0 \%$ & $100 \%$ & $0 \%$ & $0 \%$ \\
\hline$q^{\prime \prime}$ & $8.7 \%$ & $0 \%$ & $91.3 \%$ & $13.6 \%$ & $86.3 \%$ & $0 \%$ \\
\hline
\end{tabular}

Table 2: In Model 2 environmental stress only impacts the frequency of female and hermaphrodite progeny of selfing hermaphrodites. Data were compiled from Kanzaki et al., 2017; Shakes et al., 2011; Tandonnet et al., 2018; Zuco et al., 2018; A. Pires-daSilva, personal communication.

\begin{tabular}{|c|c|c|c|c|c|c|}
\hline & \multicolumn{3}{|c|}{ NO STRESS } & \multicolumn{3}{|c|}{ STRESS } \\
\hline & $\sigma^{\prime \prime}$ & $q^{\prime \prime}$ & q & $\sigma^{\prime \prime}$ & $q^{\prime \prime}$ & 우 \\
\hline$\sigma^{\prime \prime} \times$ o & $18 \%$ & $82 \%$ & $0 \%$ & $18 \%$ & $82 \%$ & $0 \%$ \\
\hline$\sigma^{\prime \prime} \times \sigma^{\prime \prime}$ & $100 \%$ & $0 \%$ & $0 \%$ & $100 \%$ & $0 \%$ & $0 \%$ \\
\hline o" & $8.7 \%$ & $0.2 \%$ & $91.1 \%$ & $13.6 \%$ & $80.1 \%$ & $6.2 \%$ \\
\hline
\end{tabular}




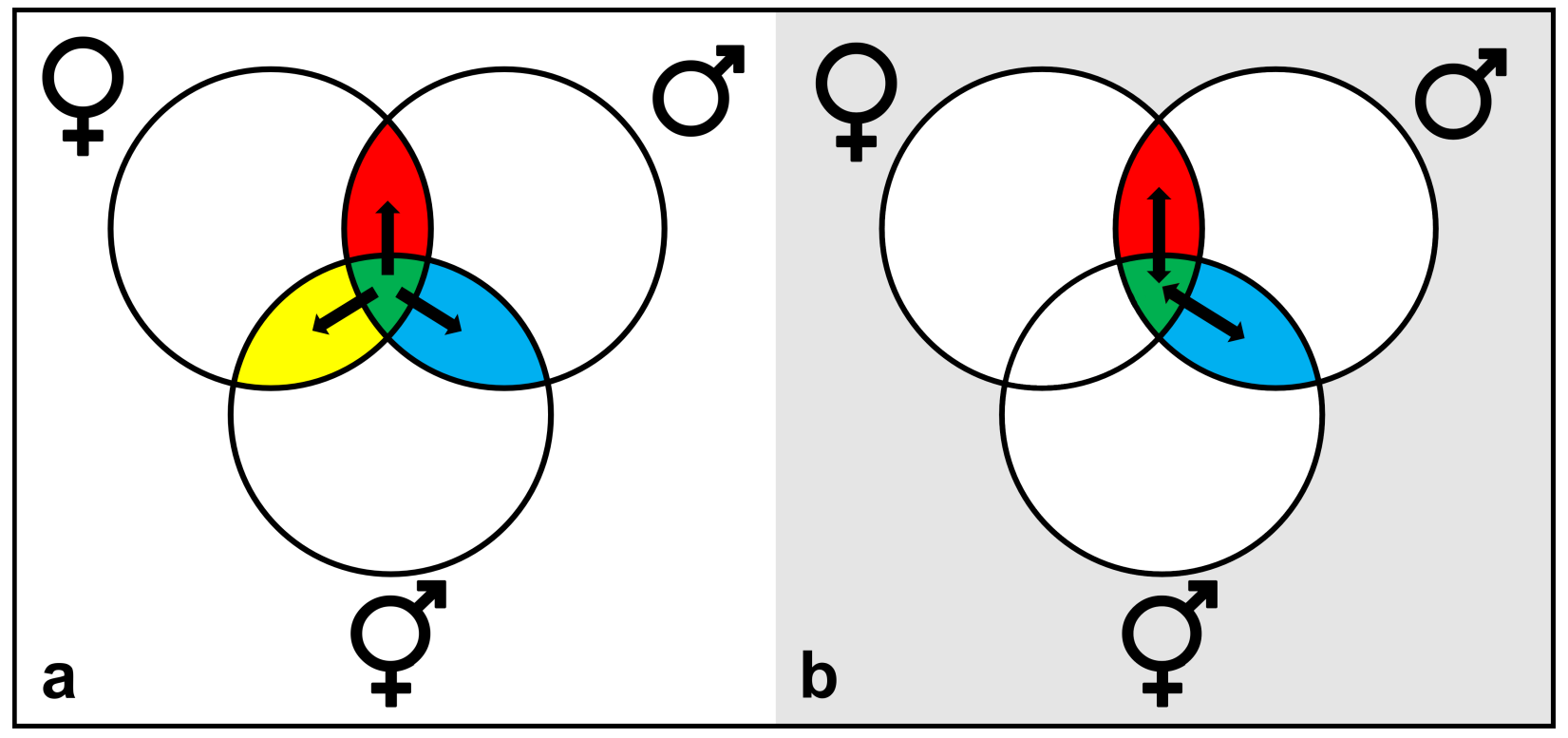

Figure 1: Intersections of the diagram represent mating systems with that combination of sexes. (a) Trioecy (green) is unstable and tends to collapse to a two-sex mating system. (b) In nematodes it has been proposed that trioecy (green) can be used as a temporary mating system in the transition between dioecy (red) and androdioecy (blue). 


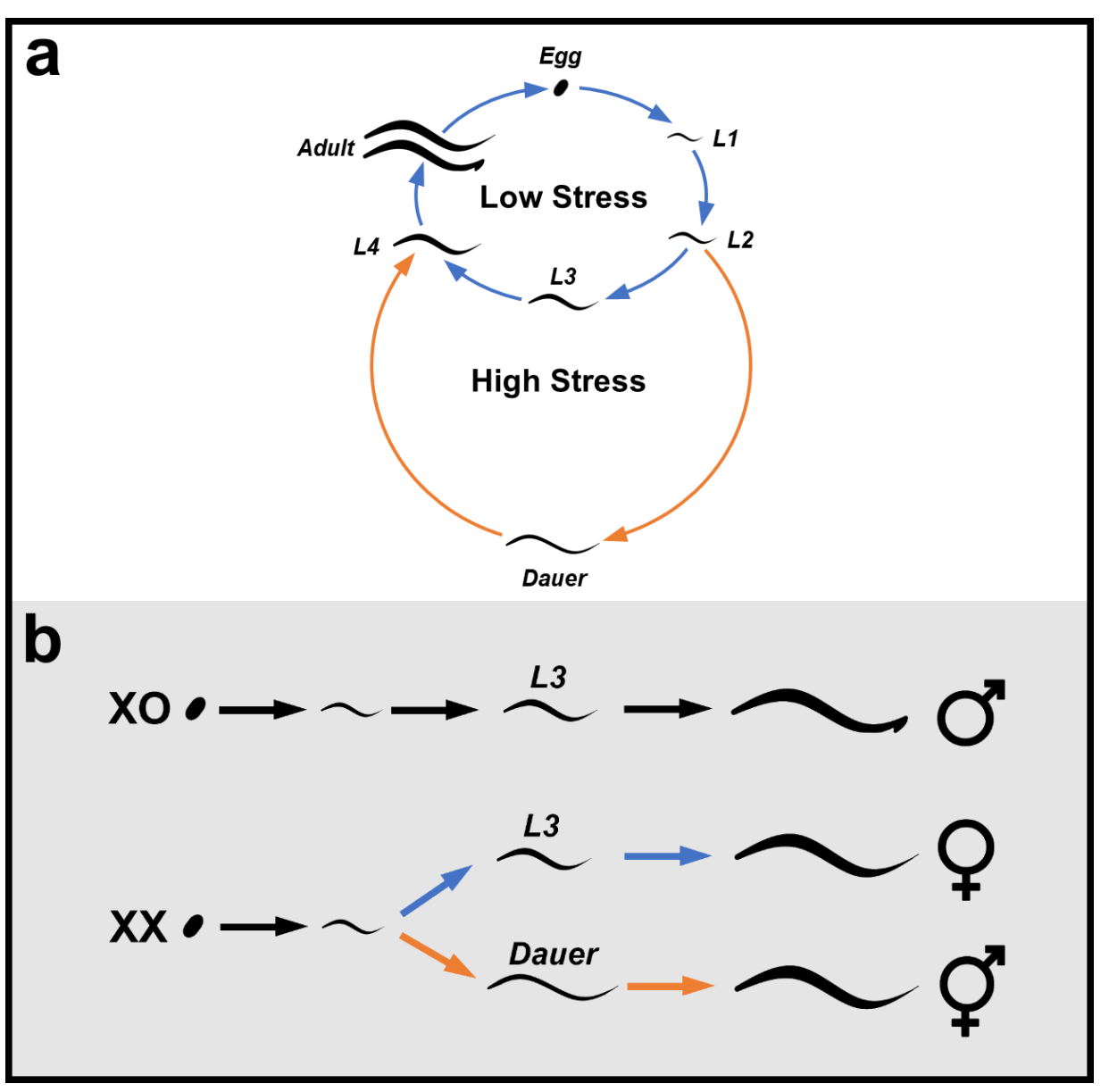

Figure 2: (a) Nematodes have a conserved life cycle of four larval stages. Many nematodes have an additional larval stage, the dauer, that is entered when stress is high. (b) In A. freiburgensis XO individuals always develop through the L3 stage and become males. XX individuals can develop one of two ways depending on the maternal stress conditions. XX worms produced by mothers under low stress (blue) pass through L3 and develop into females. XX worms produced by mothers under high stress (orange) pass through dauer and become hermaphrodites. 


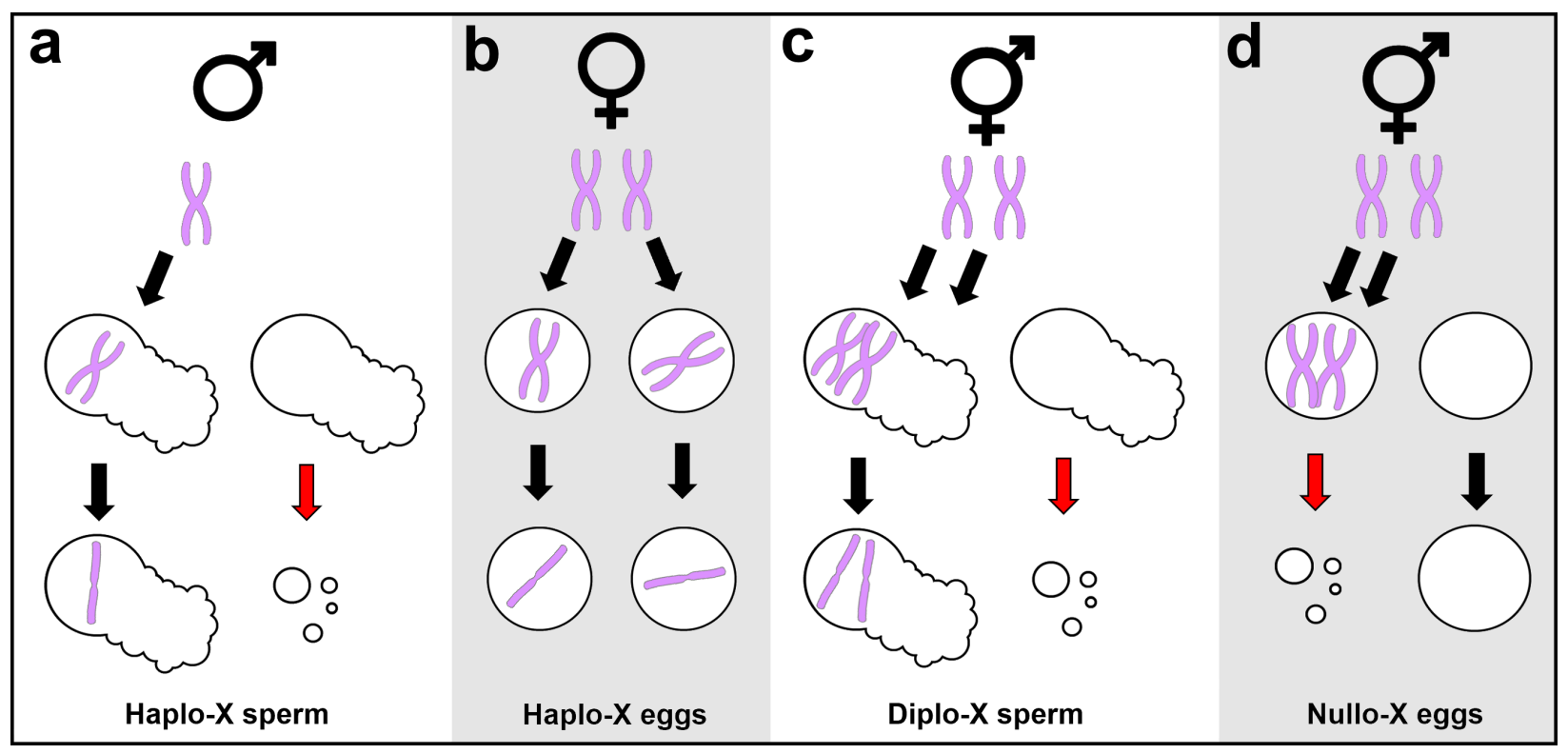

Figure 3: Meiosis in A. rhodensis produces unexpected gametes in males and hermaphrodites. The gametes formed, as well as a relatively high frequency of nondisjuction, probably contribute $A$. freiburgensis sex ratio data that are highly divergent from what would be expected. 


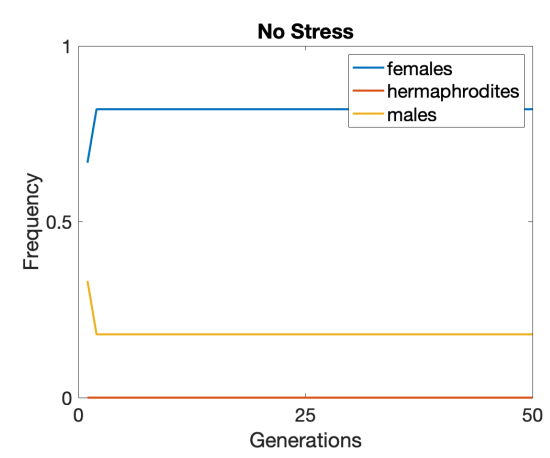

(a)

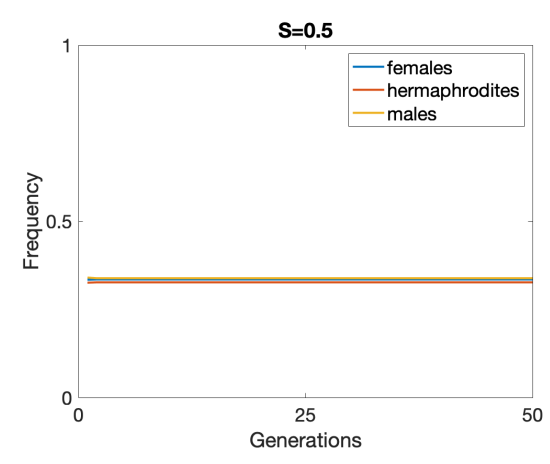

(c)

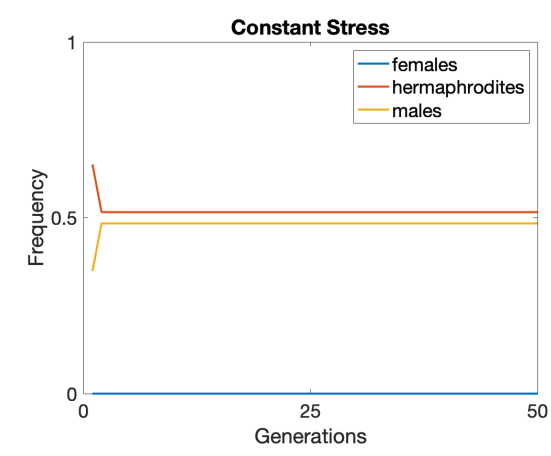

(b)

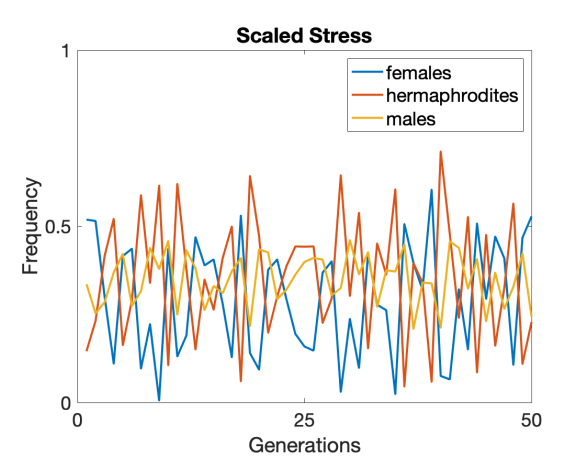

(d)

Figure 4: In Model 1 the mating system is determined by environmental stress. When there is no stress (a) the population is composed of females and males and when there is high stress (b) the population is composed of hermaphrodites and males. With (c) intermediate stress trioecy is stably maintained and with (d) fluctuating random stress all three genders are maintained in the population. Here, $\theta_{F}=0.2, \theta_{H}=0.6$, $d=0.01, P_{0}=0.33, Q_{0}=0.33$ and $R_{0}=0.33$. 

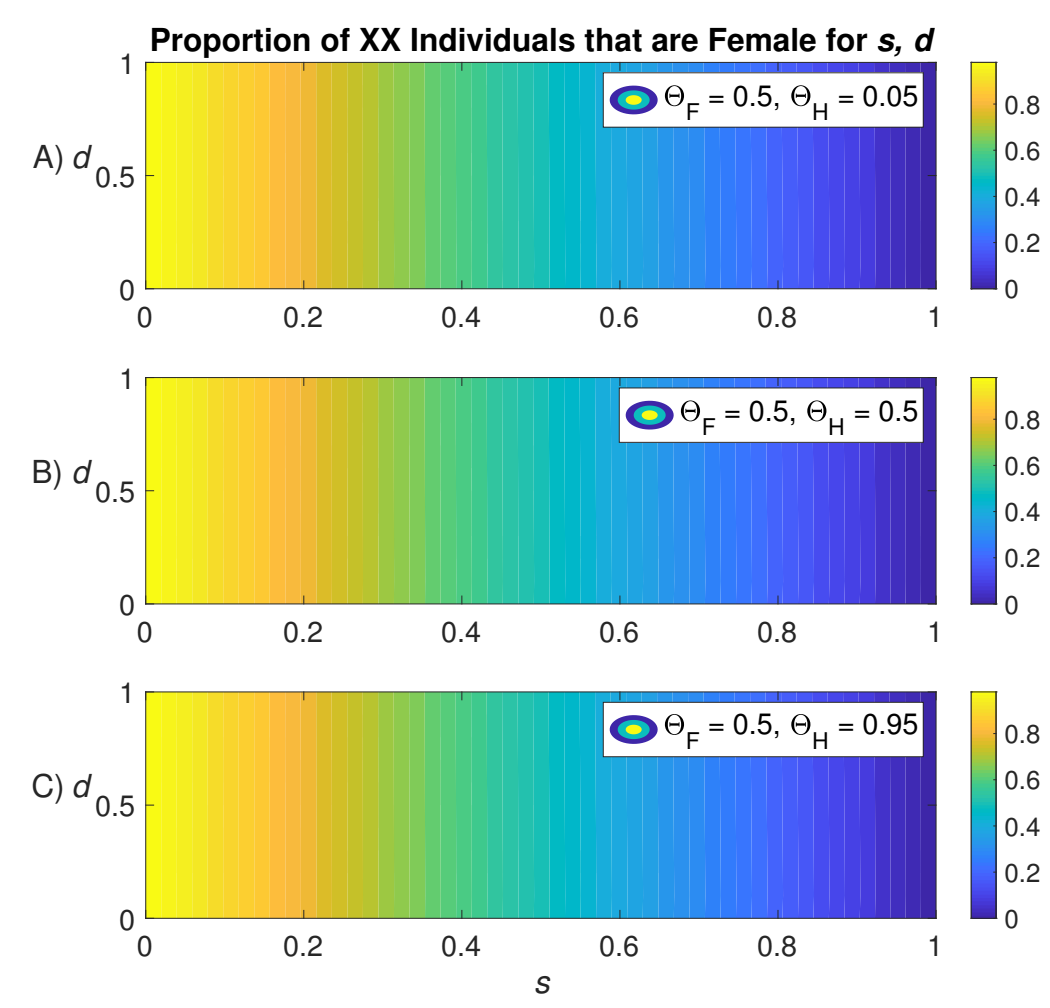

Figure 5: In Model 1 the proportion of females relative to hermaphrodites is strongly determined by $s$. Here, the proportion of females is calculated as $P /(P+Q)$. Iteration over the full range of combinations of $s, d, \Theta_{F}$ and $\Theta_{H}$ is shown in the Supplemental Materials. 


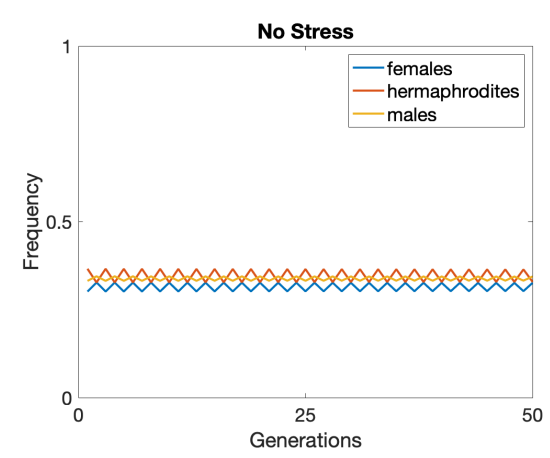

(a)

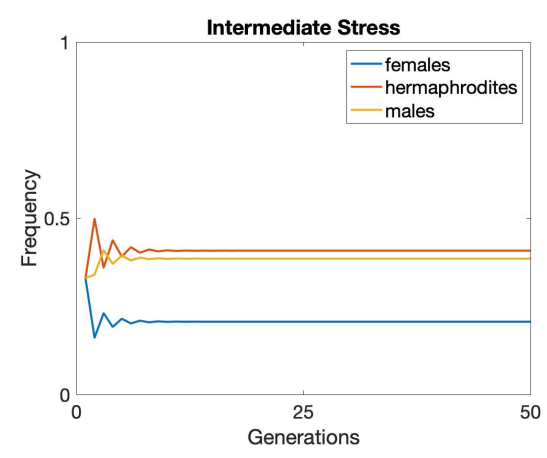

(c)

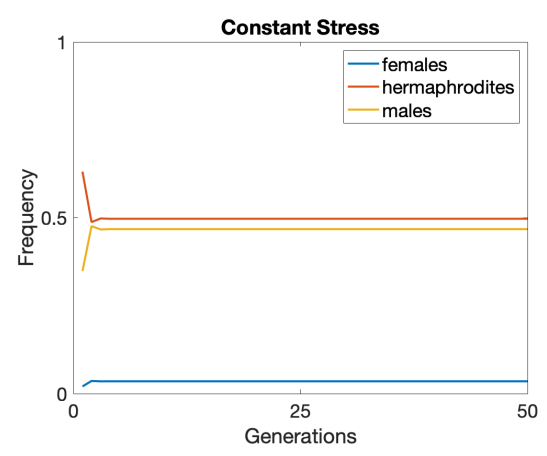

(b)

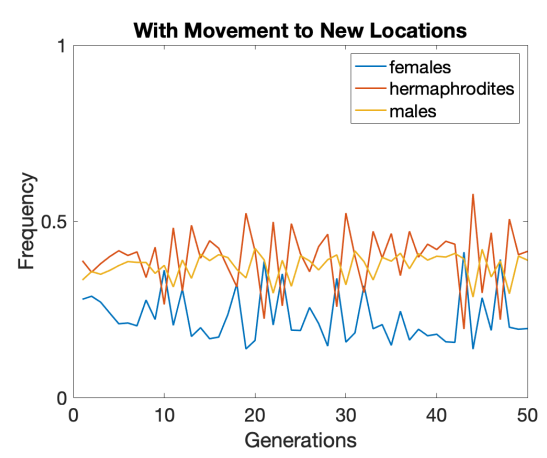

(d)

Figure 6: In Model 2 females, males and hermaphrodites are maintained in populations that experience (a) no stress; (b) constant stress; (c) intermediate stress; and (d) varying stress. For each generation in (d), a random value between 0 and 1 was chosen to represent the stress in the environment at generation $n$. Here, $\theta_{F}=0.2, \theta_{H}=0.6, d=0.01, P_{0}=0.33, Q_{0}=0.33$ and $R_{0}=0.33$. 
bioRxiv preprint doi: https://doi.org/10.1101/862664; this version posted December 3, 2019. The copyright holder for this preprint (which was not certified by peer review) is the author/funder, who has granted bioRxiv a license to display the preprint in perpetuity. It is made available under aCC-BY-NC-ND 4.0 International license.

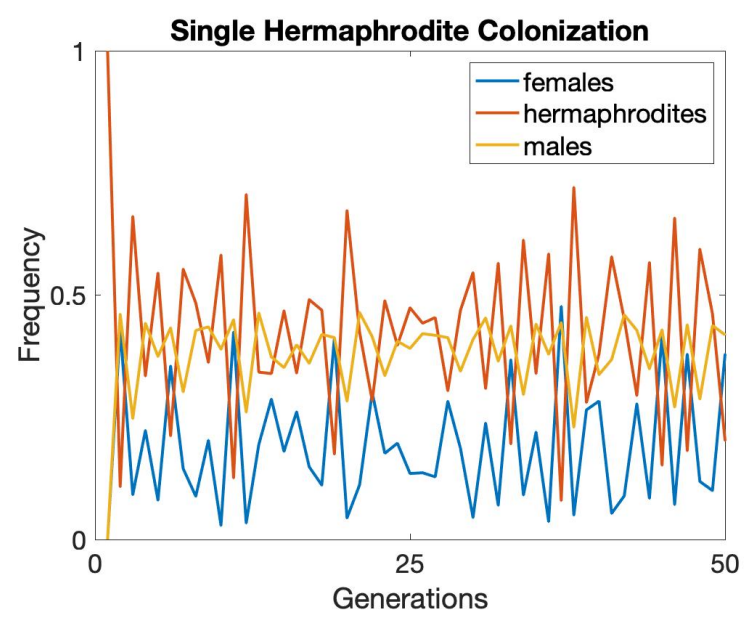

Figure 7: A single hermaphrodite can produce a trioecious population under the conditions of Model 2.

Here, $\theta_{F}=0.2, \theta_{H}=0.6, d=0.01, P_{0}=0.33, Q_{0}=0.33$ and $R_{0}=0.33$. 


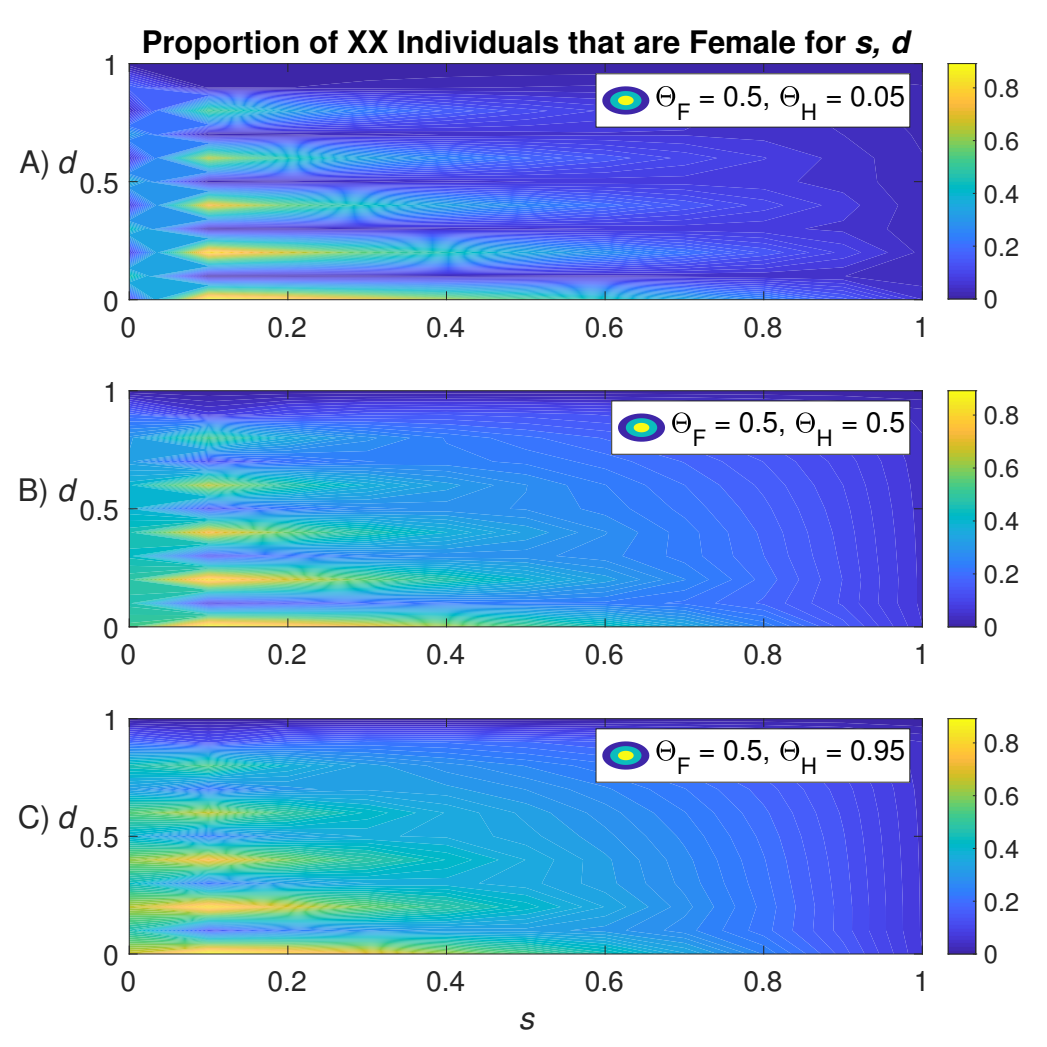

Figure 8: In Model 2 the proportion of females relative to hermaphrodites is nonlinear across the range of $s$ and $d$. Here, the proportion of females is calculated as $P /(P+Q)$. Iteration over the full range of combinations of $s, d, \Theta_{F}$ and $\Theta_{H}$ is shown in the Supplemental Materials. 